\title{
Modelling Design of Multiphase Bubble-Bed Reactors for Advanced Food-Industry Applications
}

\author{
J Zahradnik ${ }^{1}$ \\ M Ruzicka ${ }^{1}$ \\ J Markos ${ }^{2}$ \\ $\mathrm{J}$ Teixeira ${ }^{3}$ \\ S Generalis 4 \\ N Thomas ${ }^{5 *}$ \\ ${ }^{1}$ Institute Chemical Process Fundamentals, Prague, Czech Republic \\ ${ }^{2}$ Univeristy of Technology, Bratislava, Slovakia \\ ${ }^{3}$ University of Minho, Braga, Portugal \\ ${ }^{4}$ Aston University, Birmingham, England \\ ${ }^{5 *}$ FRED Ltd - Aston Science Park, Birmingham B7'4BJ UK (neale@thomas.net)

\section{Columns / Loops \\ Hydrodynamics \\ Fermentation \\ CFD} \\ Food-Industry.
}

An EC project (IC15-CT98-0904 / PL979021) under this title commenced November 1998 courtesy of Dr Jindrich Zahradnik, sadly since deceased. In dedication to his memory overviewed here are contributions from the four partners whose lead investigators appear as authors (plus coordinator as corresponding author) with principals and researchers recognised in cited literature. A website (www.copernicus.aston.ac.uk) has been launched to disseminate major individual components and collaborations facilitated by study exchanges, also envisaged exploitation by industries. Drawing on this material we outline partners' established expertise and its unification under EC umbrella funding. To avoid confusion on due credit for contributions, references are designated by first letters of the above-named authors. At risk of appearing to favour ones' own wares, we humbly refer readers to our cited papers for contextual commentaries.

\section{Introduction}

Prior liaison as projects under the auspices of Royal Society / Czech Academy Sciences support provided the platform for unifying complementary expertise from the lead and corresponding authors, the former in equipment evaluation and interpretation, the latter in flow fundamentals and implementations for plant process biotechnology. This earliest collaboration emphasised incorporation of physicochemical realism into established understanding achieved with simple air-water systems, for example viscous solutions [Z1] and electrolytic solutions [Z2] for their effects on operational performance from bubble size amplification / inhibition respectively by suppression of fragmentation and coalescence, These first successes were consolidated with attacks on the hoary old problem of collapse of quiescence in uniformly base-sparged bubble beds, initially within the classical framework of pattern-parameterised transition [Z3], subsequently more in terms of fundamental factors like bubble formation and release [R0]. The 
last study in effect launched our present project, at least its component on hydrodynamic fundamentals pursued with special attention to the roles of gas entry conditions [R2] and couplings for near field behaviour and modal interpretations [R1], also for new representations of synergistic bubble interactions via nonlinear paradigms [R3]. It also afforded access to reconciliation of contentious issues in applicable scalings for regime transition in real bubble columns (R4) and consolidation of an old speculation that instability inception in bubble columns might be analogous to that manifested in thermal convective transition of uniphase fluids heated from below [R5].

These basic advances are providing useful new theoretical foundations not only for better interpretations of experimental data and its application by scaling correlations to bioreactor performance evaluation but also for efficacy validation of "CFD" codes on which some sector hopes have been pinned as avenues to improved and cheaper protocols in exploring configurational and operational options. Our project entails elements of all these aspects, including physical performance evaluations / correlations for circulation [M1] and oxygen transfer [T2] in airlift loops and bend effects [M3] to pilot scale plus pulsed delivery options [M4] overcoming inhibition in viscous broths, also mass-loading impairment of transport dynamics in floc-stabilised cultures [T3], respectively as precursors for implementation in real fermentations of citric acid [M2, M5] and for ethanol [T1]. CFD aspects are included via a familiarisation / implementation training exercise [G1] using FLUENT chosen as a globally established product with sustained support services. This aspect is anticipated to be of end-user interest principally as a prospective teaching / training tool for improved insight by bioprocess engineers, many of whom from biochemical backgrounds lack firsthand familiarity with intricate complexities [G2] of multiphase turbulence in simple fluids, never mind the challenging constitutions of real broths.

\section{Physical Basics}

The major determinant of microscale behaviour resides in hydrodynamic coupling between adjacent orifices in perforated plate spargers, indeed is of crucial importance for whether or not a uniform bubbly layer can be formed. To elucidate the nature of interference between orifices fed from a common gas plenum below the plate, experiments were performed in which bubbling mode was identified by signature in local pressure records. Two interacting orifices [R1] deliver synchronous bubbling regimes at low and high flow rates, these limiting behaviours separated by a wide range of asynchronous regimes. Important operational parameters include barriers in the plenum and in the liquid, orifice spacing, water height and column diameter, all of which affect the stability thresholds. Special attention was paid to the lower synchronous regime in evaluating bubbling from multiple orifices [R2] for which families of modes were identified ranging from completely asynchronous, via partially synchronous to completely synchronous. Interactions between orifices on both the gas and liquid sides were explored for their significance and main factors were identified extending also to role of symmetry in orifice distribution over the plate in establishment of resonant bubbling conditions.

Mesoscale investigations were done on interacting bubbles as a step towards understanding some basic features of idealised cluster dynamics. Linear chains of rising bubbles were explored theoretically [R3] using a force-law model akin to mechanical mass-spring damper models for elucidation of their behaviour over representative Reynolds numbers. Three forces (buoyancy, drag, inertia) were taken to determine chain response via local and non-local interactions with two different assumed boundary conditions (free-end and fixed-end). Free-end chains tend to split into smaller links that subsequently interact and produce a highly fragmented long-time structure, typically via merger, separation, pairing, re-pairing and oscillation. Fixed-end chains can sustain uniform spacing and they rise faster than isolated bubbles, also they can sustain 
propagating concentration waves but at low Reynolds number the uniform spacing yields to chaotic behaviour. All results produced by the model compare plausibly with published data.

Macroscale modelling ideas have been assessed for merit as descriptions of conditions prevailing at collapse of homogeneous regime with increasing voidage. They comprise kinematic and dynamic approximations by which is meant the former assumes equilibrium dynamics as algebraic closure for continuity whilst the latter admits coupled perturbation dynamics. The kinematic model provides a simple transparent representation of regime transition [R4] in which homogeneous closure is made via Darwinian drift that also furnishes a simple exact formula for voidage dependence of fractional deficit in bubble slip speed. In heterogeneous regime, the coupling closure was assigned in a simple formal way consistent with Zuber-Findlay classical drift-flux formulation. Emulating traditional practice in representation of laminar-turbulence intermittency, regime transition is approximated by a smoothed intermittency function. The resulting model has five parameters each with unambiguous physical meaning and each enumerable from experimental data: namely two terminal bubble velocities, the bubble drift coefficient, the Zuber-Findlay constant and an intermittency factor. Its resulting formulae describe voidage-gas flow rate dependence separately in the homogeneous, heterogeneous and transition regimes as well as predicting critical conditions for homogeneous regime instability and maximum voidage. Experiments conducted with four different air-water bubble columns show excellent match between predictions and these data, also published data and linear stability analysis.

The macroscale dynamic idea postulates a simple analogy with thermal convective instability, being also a subcritically inactive horizontal layer subjected to buoyancy flux introduced from the bottom boundary. In classical convection the layer becomes unstable to disturbances above a critical Rayleigh number dependent on Nusselt and Prandtl numbers plus aspect ratio. In bubbly convection the layer is a subcritically passive gas-sparged liquid that breaks into large-scale motion. On this basis it is tempting to assign equivalent numbers such that regime transition is captured by analogy. Elucidating experiments were done in cylindrical containers to avoid "corner" anomalies and a stability criterion was obtained [R5] very much in line with expectations from established thermal findings. Indeed homology between the governing equations can be demonstrated formally in the absence of bubble inertia and bubble slip speed, similarly also for sedimentation of dispersed particles.

\section{Biochemical Engineering}

Fermentation for citric acid by A. niger is interesting [M2] because initially Newtonian aqueous liquid increasingly exhibits non-Newtonian pseudo-plasticity. Fermentation for gluconic acid [M5] entails complexities of three-phase GLS phenomenology with spherical pellets of fungi dispersed in bubbly aqueous liquid. Production of citric acid is achieved only in growth conditions where sacharidic nutrient and dissolved oxygen are metabolised to product and for biomass, with outcome sensitive to dissolved oxygen deficicit but not to $\mathrm{pH}$. Production of gluconic acid is delivered in both growth and stationary conditions, both oxygen and $\mathrm{pH}$ sensitive but the former entailing sterility and complex media also two-phase becoming three-phase whereas the latter avoids these costly complications. Continuous ethanol production using flocculent yeast strain provides a three-phase system with high solid fraction with important rheological response [T1]. A unit was implemented for configurationally versatile column and loop fermentations and two loop reactors (one internal, one external) were also installed.

Evaluations using Aspergillus niger fermentations and related transport diagnostics were as follows. For liquid circulation velocity, long-established as a benchmark indicator for loop 
reactors, a simple cheap method was implemented [M1] using a neutrally buoyant particle with a high magnetic permeability. The results were similar to those obtained using dissolved tracers. Comparative studies of citric acid fermentations [M2] failed to elucidate any configurational sensitivity although bubble separators have long been known to determine carry-over into loop downcomers, with obvious adverse effects on intensity and stability of the circulation. Accordingly an evaluation was done [M3] to assess its impact for standard measures like downcomer holdup, circulation velocity, mixing intensity and gas circulation. Gas-liquid transfer is often a limiting factor in aerobic fermentations, exacerbated by elevated viscosity associated with high biomass densities compounding their high oxygen demand. We found [M4] that periodic pulsing of gas flow increases dissolved oxygen concentration in citric acid fermentations, an effect attributed to more complete utilisation of oxygen in small foam bubbles. The pulsing strategy was optimised to maximise unsteady gas hold-up and dissolved oxygen and demonstrated that efficient operation can be maintained even in highly viscous broths. Stationary fermentation of gluconic acid was exploited [M5] to assess oxygen utilisation in bioconversion of glucose to gluconic acid and thence by difference also to estimate that taken up for biomass growth. The specific rate of Gluconic acid production depended strongly on air rate not just for increased oxygen availability but also intensified circulation and mixing. Whilst biomass concentration boosted production rate, its adverse impact on oxygen transfer significantly suppressed production, in line with Michaelis-Menten expectation. However the rigid pellets were seen to intensify bubble coalescence and break-up, a compensating factor that has not been routinely reflected in published performance correlations.

Evaluations using ethanol fermentations [T1] and related transport diagnostics were as follows. Mass loading impairment of transport dynamics in floc-stabilised cultures was assessed using measurements of riser gas hold-up, downcomer and riser liquid velocity, circulation and mixing times. Calcium alginate beads simulating yeast cell flocs were used as solid phase. Riser gas holdup decreased with solids loading, behaviour attributed to coalescence-induced slip-speed enhancement and it increased with increasing solids density. The effect was more pronounced in the ILR and solids density had negligible influence on circulation and solids distribution in the ELR. Whilst ELR circulation was satisfactory at higher solids loading, ILR circulation eventually ceased due to bend throttling. Circulation velocity increased similarly with superficial gas velocity in both reactors, both going as a typical power law with weak influence of solids loading. Solids loading also had little influence on circulation time dependence on riser superficial gas velocity, increasing it slightly in the ILR and decreasing it slightly in the ELR. Mixing time increased slightly with solids fraction in both reactors. A correlational model was produced and demonstrated for these GLS 3-phase reactors with high loading of low-density solids. Gas sparging plates were also used to show insignificant influence on ELR performance measures. A modified Zuber-Findlay model was used to characterise riser gas hold-up and an overall energy balance to retrieve the different riser superficial velocities in the ILR and ELR [T2, T3]. The model captures main elements of aforementioned behaviour, in particular slip-speed increase is attributed to coalescence driven by bubble-bubble interactions, whilst solids loading respectively decreased / increased circulation in the ILR / ELR.

\section{Computational Modelling}

Whilst industrial CFD codes cannot furnish the realistic and reliable representations needed for practitioner assurance as operational toolkits, they do at least provide an opportunity platform as introductory familiarisation aids and could be especially valuable for process engineers entering the profession from biochemical speciality backgrounds. Their major strength in this respect is painless accessibility to plausible realisations of the complex flow patterns and events that are mainly responsible for serious shortcomings in the grossly oversimplified treatments traditionally 
employed for biochemical performance estimation. Recent endeavours toward this goal are outlined below and the interested reader should refer to cited outputs for access to the specialist contextual literature.

To ensure ready availability for prospective implementers (and to avoid wheel reinvention) we chose to adopt a widely available proprietary code, specifically the FLUENT software - not for any special reason by comparison with competing products, simply for its global supply-support services. The code employs bog-standard formulations for k-epsilon modelling of turbulence and algebraic-slip mixture modelling for bubbly fluids, all as specified in Fluent Inc literature. To date attention has been focused on boundary conditions representative of bubble columns rather than loops, the challenges for columns being more than enough to keep the researcher busily occupied in meshing for efficient convergence, not to mention the computer (a bog-standard PC) running red-hot especially in going from 2D to 3D geometries! Velocity fields computed for these cases exhibit adjustment from symmetric to asymmetric [G2] as preferred prevailing mode, probably a generic response for confined buoyant plumes and not needing any special explanation for two-phase fluids. The 2D profile is also shown [G1] to compare favourably with experimental results, implying that whilst individual 3D realisations are asymmetric their longterm ensemble retains symmetry.

As a first feel for (bio)particle movements in these fields the importance of fractional density excess was assessed for nearly neutral trajectories and those with small excess, both migrating downwards within wall adjacent down-flow and the heavier species of course being less wellsupported in transient central upflows. Both species manifest evidence of vortex hold-up which has been the subject of much attention in the fundamental literature on multiphase transport (refer to cited papers for references). However it is clear that computing individual realisations for their ensemble statistics (the meaningful measure for long-term processing performance) is an impracticable proposition, indeed as has been long recognised by the industial multiphase community from whom originally came the motivation for mixture models like the one used here for the bubbly fluid motions. In this spirit then some first steps have been taken toward incorporation of a solid phase mixture model, thus far using a slip-speed advection-diffusion approximation with only one-way coupling (ie no mass-loading feedback on the bubbly mixture motion). First results portray this averaged evolution for an (idealised) introduction across the top-left half boundary and shows the predominantly downward migration driven by density excess resides preferentially along the wall as seen in the individual trajectory realisations. However some material is transported across the column axis where it also is attracted to the opposite wall during its downward drift. With the long-term trend here toward sedimentary stratification it is clear that the chosen parameters employed are unrepresentative of conditions suited to bioprocessing - but then it was precisely this kind of adaptive learning exercise for which the activity was intended. The next stage will focus on advancing and proving the transport model to which will be added simple biokinetics, simple but sufficient to support qualitative illustrations of nutrient uptake, biomass accumulation and product generation.

\section{Retrospection}

Viewed as an EC Framework 4 Thematic Network activity the project has undoubtedly been successful in catalysing several study stays broadening the experience and skills of trainee researchers, probably also in opening the eyes of established professionals as to the complexities of components beyond individual familiarity horizons. Measured in terms of outputs the project has also succeeded in raising sophistication of RTD activities by individual participants, not least from new opportunities for interactions with sector leading peers and dissemination outlets for contributions - all being important components for confidence and 
competence of all participants as sector specialists. However, in terms of delivering these capabilities as new commercial consolidations we have long ways to go. Whilst better basics, confirmed correlations and verified virtualities all assist in background building for future implementation and exploitation, we could not yet claim to have achieved worthwhile movement toward adoption by prospective end-point beneficiaries. However, with perseverance we may yet prevail and our website launched and supported gratis courtesy of Aston University is the backbone to this endeavour.

\section{Literature}

[Z1] Snape JB, Zahradnik J, Fialova M, Thomas NH. 1995. Chem Eng Sci 50, 3175-3186.

[Z2] Zahradnik J, Fialova M, Kastanek F, Green KD, Thomas NH. 1995. Chem Eng Res Des 73, 341-346.

[Z3] Zahradnik J, Fialova M, Ruzicka M, Drahos J, Thomas NH. 1998. Chem Eng Sci 52, 38113826.

[R0] Ruzicka MC, Drahos J, Zahradnik J, Thomas NH. 1997. Int J Multiphase Flow 23, 671-682.

[R1] Ruzicka M, Drahos J, Zahradnik J, Thomas NH. 2000. Chem. Eng. Sci. 55, 421-429.

[R2] Ruzicka M, Drahos J, Zahradnik J, Thomas NH. 1999. Chem Eng Sci 54, 5223-5229.

[R3] Ruzicka M. 2000. Int J Multiphase Flow 26, 1141-1181.

[R4] Ruzicka M, Zahradnik J, Drahos J, Thomas NH. 2001. Chem Eng Sci - accepted.

[R5] Ruzicka M, Thomas NH. 2001. In preparation.

[M1] Klein J, Dolgos O, Godó S, Blazej M, Markos J. 2000. Chem Pap 54, 456-466.

[M2] Klein J, Godó S, Báles V. 1999. Bulgarian Chem Comms 34, 547.

[M3] Klein J, Godó S, Dolgos O, Markos J. 2000. J Chem Tech Biotech - accepted.

[M4] Godó S, Klein J, Polakovic, M, Báles V. 1999. Chem Eng Sci 54, 4937-4943.

[M5] Klein J, Rosenberg M, Markos J, Dolgos O, Kroslák M, Kristofíková \&Lapos;. 2000. Bioproc Eng - submitted.

[T1] Domingues L, Lima N, Teixeira JA. 1999. Biotech Bioeng 64(6), 692-697.

[T2] Freitas C, Fialová M, Zahradnik J, Teixeira JT. 1999. Chem Eng Sci 54, 5253-5258.

[T3] Freitas C, Fialová M, Zahradnik J, Teixeira JT. 2000. Chem. Eng. Science 55, 4961-4972.

[G1] Cartland Glover GM, Generalis SC, Thomas NH. 2000. Chem Paps, 54(6a), 361-369.

[G2] Cartland Glover GM, Generalis SC, Thomas NH. 2000. CHISA, Prague. 